\title{
Model-based Integration of Tools Supporting Automatable IT Service Management Processes
}

\author{
Ingo Pansa ${ }^{1}$, Philipp Walter ${ }^{2}$, Sebastian Abeck ${ }^{1}$ \\ Cooperation \& Management \\ Karlsruhe Institute of Technology (KIT) \\ 76131 Karlsruhe \\ ${ }^{1}\left\{\right.$ pansa $\mid$ abeck \}@kit.edu ${ }^{2}$ pwalter@cm-tm.uka.de
}

\author{
Klaus Scheibenberger \\ IT Infrastructure and Services \\ Karlsruhe Institute of Technology (KIT) \\ 76131 Karlsruhe \\ scheibenberger@kit.edu
}

\begin{abstract}
Shifting towards process-oriented approaches for IT Service Management (ITSM) is a rather evolutionary than a revolutionary step, enabling IT service providers to automate parts of their management activities. Therefore the existing management tools have to be integrated into an architecture that is aligned with the ITSM processes. The challenge in achieving this lies in the decoupling of the processes from the functionality offered by a heterogeneous tool chain. Applying serviceorientation to integrate the existing management tools can help to solve this issue. Existing work within this field hardly discusses the necessary concepts in integrating management tools from a management process perspective but mainly focus on technical details such as web service-based integration making it hard to adapt the proposed solutions to scenarios other than targeted. To address this situation exemplified by ISO/IEC20000's definition of Incident Management Process, we present an approach for designing management services independent of concrete tools. The interfaces of the management services are designed with respect to requirements that can be derived from management standards and thus can be reused in management processes that conform to ISO/IEC20000.
\end{abstract}

Keywords- ISO/IEC20000, IT Service Management, Web Service, Model-based development approach

\section{INTRODUCTION}

Nowadays business activities strongly rely on services implemented by systems that are based on Information Technology (IT). Thus, IT-based services (IT services) need to be both available and reliable in an environment in which the requirements for managing these services are constantly increasing. The most recent efforts have ultimately led to the standardization of process-oriented IT Service Management (ITSM) approaches such as ISO/IEC-20000:1-2005 [1] which was largely inspired by the Information Technology Infrastructure Library (ITIL) [2]. This standardized approach to structuring the organizational concerns related to IT service management allows parts of the management processes to be automated, which was shown to improve the quality of the observed management activities.

From the view of an IT service provider, the IT services are composed of heterogeneous networks as well as different system and application technologies making it likely that different management tools or even complete management systems are responsible for the various management related aspects. In a typical e-mail service, for example, a couple of network components (such as switches, routers and firewalls) interconnect the various server systems running for instance a directory system, a mail transport system or a mailbox system. Each of these different components is probably managed with a variety of management tools. An overall management process usually gathers information from each of the individual management tools. This scenario not only hampers the adoption of process-oriented approaches in general, but also hinders the automation of parts of these processes in particular, as the tools were not designed to consider overall organizational aspects such as the sharing of management information. Therefore, possibilities have to be investigated to add functional interfaces to existing tools to close this gap between the organizational aspects of processes and technical aspects of management tools. The web service-based Serviceoriented Architecture (SOA) as an integration architecture that supports automatable processes proves to be a pragmatic solution to many of the integration problems arising.

However, using web services for a service-oriented integration of existing software systems increases complexity by introducing an additional layer that acts as a middleware. To tackle this additional complexity, a systematic approach leading from problem statement to engineered solution is needed, considering the various aspects of software engineering. A systematic approach based on formal models can support the engineering of management architecture in that both the technological concerns regarding adapter construction (such as mapping the APIs of existing tools to web service adapters) and the requirements that are derivable from a management standard can be captured and provided to domain experts. For a first contribution to such an approach, this paper presents initial results towards a conceptual model of ISO/IEC20000-1:2005. We show how such a model can help to design management services that support the integration of existing tools and the alignment with standardized ITSM processes likewise.

\section{RELATED WORK}

One rationale for investigating ways of tackling management complexity is grounded in the fact that human beings tend to structure complex activities by grouping repeatable activities. Over the last decades, efforts to find 
solutions have led to process-oriented management frameworks. Process-oriented management frameworks for managing the various concerns related to IT service production have been in the spotlight since Great Britain's Central Computer and Telecommunication Agency (CCTA) published a collection of best practices named Information Technology Infrastructure Library (ITIL) [2] in late 1980's. With the upcoming success of ITIL's best practices, further processoriented approaches have recently appeared.

The most popular examples including the international standard on ITSM published by the ISO (ISO/IEC200001:2005 [1]), the Microsoft Operation Framework [3], ISACA's CoBIT [4] and eTOM [5] published by the TeleManagement Forum indeed provide different suggestions on how to structure management activities. However, they all a lack guidelines on architectural construction for the purpose of tool support [12].

Because of the complexity of modern IT infrastructures, it is unlikely that one single tool can fulfill the manifold requirements inherent to process-oriented ITSM approaches. Service-oriented architecture is being promoted as a solution for many of the problems created by using multiple separated software systems within the context of organizational collaboration, which is what process-oriented ITSM figures out to be. A common definition of the term service-oriented architecture can be found in [6], stating that "Service Oriented Architecture (SOA) is a paradigm for organizing and utilizing distributed capabilities that may be under the control of different ownership domains". The promotion to adopt SOA for integrating management tools along process-oriented management approaches initially appeared in Machiraju et al. in 2004 [7], who recommended that loosely coupled approaches such as SOA should be employed to tackle the impending complexity of future management systems. Based on this assumption Mayerl, Abeck et al. present suggestions [8, 9] to integrate existing management tools along processoriented management approaches. However, the approaches presented in $[8,9]$ are rather general and do not consider formal models that support development process. For example, they do not discuss functional requirements derivable from ISO/IEC20000 or explicitly automatable parts of ITSM processes. In [19], automation is identified as one approach to tackling the challenges current management issues propose. In [20], the authors argue that service-oriented architectures will be a key part of management systems.

In [10] a prototypical implementation of a web servicebased software architecture realizing an incident management process is presented. The proposed approach focuses on the derivation of service interfaces by analyzing required process activities, but neither a formal model nor an overall development process is presented.

Schaaf and Brenner [12] present a development approach based on models leading to an approach that is similar to the one proposed by OMG's Model Driven Architecture (MDA). Their idea is based on OSI reference architecture for IT management and introduces four sub-models (function model, organization model, communication model, information model) for a ITSM architecture. Basically, they advise engineers develop tools for ITSM in a strictly top-down manner. A model-driven development methodology is proposed leading to a set of twelve different models regarding the OSI reference architecture and each of the MDA layers (CIM, PIM and PSM) is proposed. Considering the formal development of software support especially for the domain of IT Service Management, we improve the results presented in [12] by focusing on the integration of existing tools based on a formally defined metamodel.

\section{MODEL-BASED ITSM INTEGRATION}

Business-driven IT Management (BDIM) is about managing the IT layer from a business perspective. This approach utilizes models, practices and tools to quantify the dependencies between these two layers [21] and to propose solutions to tackle this challenge. This paper introduces such a model for the informally described management processes in [1] or [2] in order to perform a service-oriented integration of the respective parts of existent management tools. The longterm goal is to create domain specific languages (DSL) for different kinds of problem spaces, which enables the generation of source code based on metamodels that define syntax and semantics of the respective modeling elements. Few results have been published on the problem space of constructing an integration architecture supporting processoriented ITSM. For addressing this situation, a model-based integration approach is followed. To clearly distinguish among different types of services within the domain of ITSM, we refer to the term management service when referring to the integration of existing management tools using web services.

\section{A. ISO/IEC20000 Metamodel}

A metamodel capturing functional requirements derivable from ISO/IEC20000 has to consider several aspects. Naturally, ISO/IEC20000 does not give any technical details concerning the implementation of a management system but it at least contains concepts that can be found in various approaches for describing and modeling business processes such as the Business Process Modeling Notation (BPMN) [11] or WfMC's Workflow Metamodel [13]. Although the adoption of an existing approach for modeling processes is close at hand, we introduce a Metamodel that is based on the core concepts of the management standard: this allows us to consider a smaller set of elements. In the definition of a management process (chapters 8-13 in [1]), Entities, Participants, Activities and Policies, are mentioned. The examples are taken from the definition of the Incident Management Process, but a generalization to the remaining 12 management processes could easily be performed. Starting with these elements, further information derivable from e.g. ITIL's best practices, can be added to enrich the model, resulting in the metamodel depicted in figure 1. For instance, one of ITIL's best practices suggests that an incident record should at minimum consist of a unique identifier, a problem description and an identifier for further customer feedback, which we identified as a policy for structuring entities. 


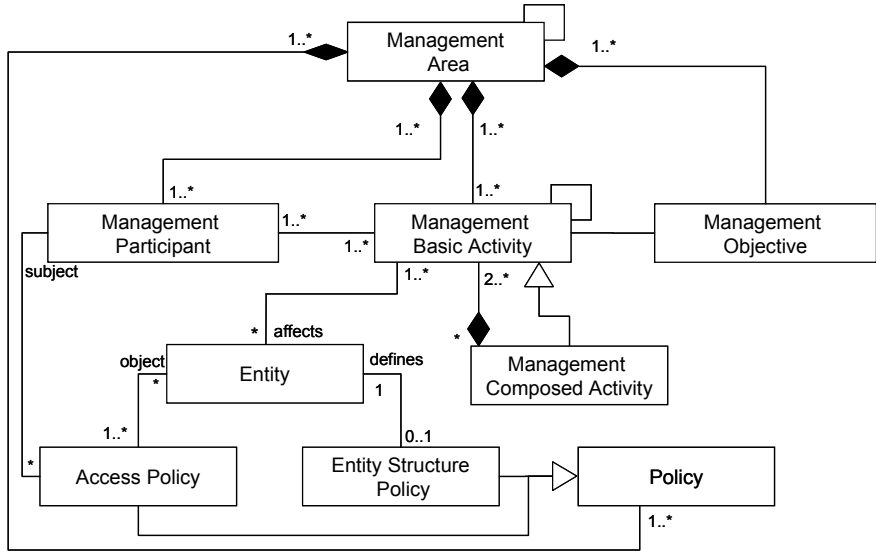

Figure 1. ISO/IEC20000 Metamodel

(Management Area) A management area represents one out of the 13 different IT Service Management processes defined by ISO20000-1:2005. Introducing a clear boundary between the different management processes enables the interfaces that separated the different concerns of integrated management architecture to be adopted.

(Management Objective) These are the core objectives that each definition of a management area describes.

(Management Participant) As the standard does not distinguish between internal and external performers of a management activity, the model introduces a generic element called Management Participant.

(Management Activity) A management activity describes the functional requirements that a specific management area offers or requires.

(Management Composed Activity) A management activity that at least contains two other management activities is considered to be a composed activity.

(Entity) An entity relates to the informational artifact upon which a management activity operates on. Typical entities include the Incident Record, Configuration Item or Service Level Agreement.

(Policy) A Policies contains the rules that can be derived from both the management standard or ITIL's best practices.

(Entity Structure Policy) As the standard specifies at least some rules and guidelines for the structural definition of management entities, a refined element is introduced.

(Access Policy) An access policy serves to define and restrict access to entites.

\section{B. Management Service Metamodel}

To enable a web service-based integration of existing management tools, different types of management services can be distinguished.

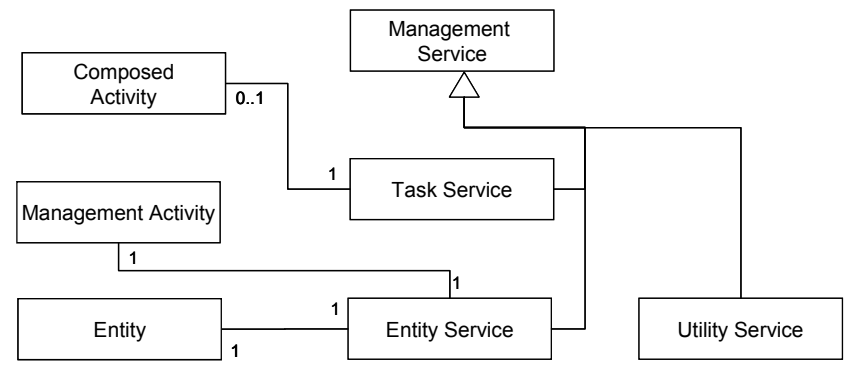

Figure 2. Figure 1 Metamodel for designing integrative management services

(Management Service) A management service describes a generic type of service that conceptualizes solution logic regarded within the domain of IT Service Management. Generally, a management service realizes the capabilities needed by management activities. As the metamodel is intended to support the early stages of a service-oriented development process, conceptual services are considered rather than physical service contracts.

(Task Service) A task service realizes basic or composed functional management activities. Further designations for this term are Process Service, Business Process service or Orchestration Service [14] or Activity Service, Capability Service [15].

(Entity Service) Entity services enable the access to the management entities. They are the building blocks of the management service architecture [14, 15].

(Utility Service) Utility services are not directly derivable from the standard requirements but that are necessary for operating the management system $[14,15]$

\section{Application of the Metamodel}

To demonstrate the usage of the models within a concrete scenario, a typical Incident Management Process that is supported by a trouble ticket tool is regarded. We decided to evaluate our approach in terms of improving incident management, as this management area is a well-known topic in last years research efforts. Starting with the definition of the management area, the participants, entity structure and access policies can be modeled according to ITIL's taxonomy. Fig 3 shows an excerpt of the investigated scenario.

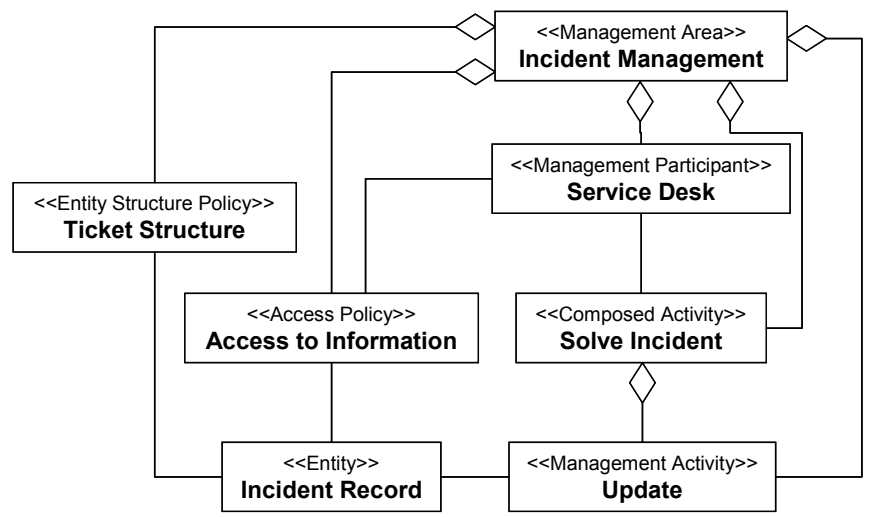

Figure 3. Instantiated model of the Incident Management process 
Having modeled the necessary parts of ISO/IEC20000 for the scenario, the management activities Solve Incident and Update can be defined according to their semantics. In our example, the management activity Update alters attributes at the trouble ticket entity, at which point an entity service is modeled. The composed activity Solve Incident is supported by several basic activities and leads to the design of a task service.

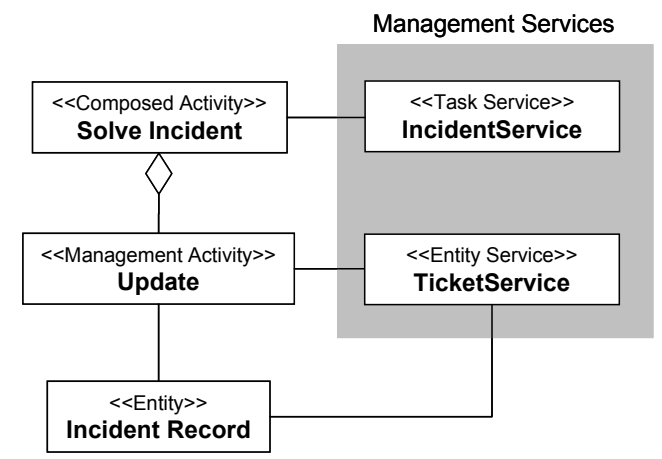

Figure 4. Web Services for integrating existing management tools

For a simple prototype, we successfully integrated an existing tool for managing trouble tickets (Mantis Bug Tracker [17]) and automated parts of the Incident Management Process using BPEL [18]. The tool was adopted through the entity service TicketService, and a part of the logic that processes an incoming incident report was implemented within the task service IncidentService (depicted in figure 4).

\section{CONCLUSION AND OUTLOOK}

This paper introduces a BDIM model [21] for the informally described management processes (e.g. in [1] or [2]) in order to perform a service-oriented integration of the respective parts of existing management tools. We propose to utilize web services for integrating management tools in order to support the automatable parts of ITSM processes.

This paper presents the initial results of the ongoing work in our research department on several aspects of the proposed service-oriented integration of existing management tools. Currently, we are exploring ways to offer engineering development tool support including our metamodel. The idea is to establish a tool kit that helps software architects to design a new or to improve existing management architecture wherefore a formalized model based on EMF currently is developed. Because the abstract syntax of the ITSM domain is formalized using techniques such as Eclipse Modeling Framework (EMF) [16] development tools specific to the problem space of integrating existing management tools into process-oriented management approaches could easily be constructed.

Our approach could also serve as input for discussing a reference model for the design of management services that could help tool vendors to create interoperable management tools by enabling service-oriented access to specific tools functionality. This would not only reduce the complexity of integrating management tools among process-oriented ITSM but also increase the comparability of management tools regarded to requirements that specific service providers have.

\section{REFERENCES}

[1] ISO/IEC, ISO/IEC 20000-1:2005: Information Technology - Service Management, www.iso.org, 2005.

[2] Office of Government Commerce (OCG): IT Infrastructure Library (ITIL) - Service Support (ISBN 0113300158), 2000; Service Delivery (ISBN 0113300174), 2001; Planning to Implement Service Management (ISBN 0113308779), 2002; Application Management (ISBN 0113308663), 2002.

[3] Microsoft TechNet: Microsoft Operations Framework 4.0, http://technet.microsoft.com/de-de/library/cc506049(en-us).aspx

[4] Information Systems Audit and Control Association (ISACA): Control Objectives for Information and Related Technology (CobiT), isaca.org/cobit

[5] TeleManagement Forum (tmf): enhanced Telecom Operations Map (eTOM), 2009.

[6] Organization for the Advancement of Structured Information Standards: Reference Model for Service Oriented Architecture, 2006.

[7] Machiraju, V.; Bartolini, C., Casati, F.: Technologies for BusinessDriven IT Management. In: Extending Web Services Technologies: The Use of Multi-Agent Approaches (Multiagent Systems, Artificial Societies and Simulated Organizations), Springer, 2005, S. 1-27.

[8] Mayerl, C.; Vogel, T.; Abeck, S.: SOA-based Integration of IT Service Management Applications. Proceedings IEEE International Conference on Web Services 2005, S. 785-787.

[9] Mayerl, C.: Tröscher, T.; Abeck, S.: Process-oriented Integration of Applications for a Service-oriented IT Management. The First International Workshop on Business-Driven IT Management, 2006.

[10] Tamm, G.; Zarnekow, R.: ITIL-konformer IT Service Support auf der Grundlage von Web-Services. 7. Internationale Tagung Wirtschaftsinformatik 2005 (Bamberg), 2005.

[11] The Object Management Group: Business Process Modeling Notation V1.1, 2008.

[12] Schaaf, T., Brenner M.: On Tool Support for Service Level Management: From Requirements to System Specifications. $3^{\text {rd }}$ International Workshop on Business-Driven IT Management, 2008.

[13] The Workflow Management Coalition Specification: Workflow Management Coalition Terminology and Glossary (WFMC-TC-1011), http://www.wfmc.org/standards/docs/TC-1011_term_glossary_v3.pdf, 1999.

[14] Erl, T.: SOA Principles of Service Design. Prentice Hall ServiceOriented Computing Series, 2008.

[15] Cohen, S.: Ontology and Taxonomy of Services in a Service-Oriented Architecture. The Architecture Journal, Volume 11, 2007.

[16] Eclipse Foundation, Eclipse Modeling Framework Project, http://www.eclipse.org/modeling/emf/, 2009.

[17] Mantis Bug Tracker, http://www.mantisbt.org/

[18] Organization for the Advancement of Structured Information Standards: Web Services Business Process Execution Language Version 2.0, 2007.

[19] Tosic V., The 5 C Challenges of Business-Driven IT Management and the $5 \mathrm{~A}$ Approaches to Addressing Them, Business-Driven IT Management, 2006. BDIM'06. The First IEEE/IFIP International Workshop on Buisness-Driven IT-Management, 2006.

[20] Machiraju V., Bartolini C. , Casati F.: Technologies for Business-Driven IT-Management, in Extending Web Services Technologies: the Use of Multi-Agent Approaches“, edited by Cavedon, L., Maamar, Z., Martin, D. and Benatallah, B., Kluwer Academic, 2005.

[21] Sauve J., Moura A., Sampaio M., Jornada J., Radziuk E.: An Introductory Overview and Survey of Business-Driven IT Management, BDIM '06. The First IEEE/IFIP International Workshop on BusinessDriven IT Management, 2006. 